\title{
A fractal load model of concrete-steel joint interface
}

\author{
Yong-Sheng $\mathrm{ZHAO}^{\mathrm{a}}$, Hao Wang ${ }^{\mathrm{b}}$, Zhi-Feng $\mathrm{LIU}^{\mathrm{c}}$ and Li-Gang CAI \\ College of Mechanical Engineering and Applied Electronics Technology, Beijing \\ University of Technology, Beijing 100124 China \\ ayszhao@bjut.edu.cn, bwhyh0917@163.com, \\ 'Izf@bjut.edu.cn, ${ }^{\mathrm{d} I g c a i 321 @ y a h o o . c o m . c n ~}$
}

\begin{abstract}
As an important connection part of machine and foundation, the machine-foundation interface have a critical effect on the working accuracy and life of whole machine. The machine-foundation interface is generated by two kinds of materials (steel and concrete), a crushed theory of asperity on concrete is proposed in this paper. This theory hold that asperity on concrete will be crushed when the load on surface go beyond the limit of stress. This paper gave the real contact area model of concrete-steel interface, the relationship of corresponding normal contact load and the real contact area of single asperity, and the different fractal dimension' influence on the contact quality of joint surface is revealed by using MATLAB simulation software.
\end{abstract}

Key words: The machine-foundation joint interface; Real contact area; Fractal theory

\section{Introduction}

Heavy-duty machine is widely applied in many industries, the machine-foundation interface as an important connecting unit, which contact characteristic directly affects work precision and service life of machine tools. The results show that the total real contact area is only $0.01 \% \sim 1 \%$ of the nominal contact area [1].

Current research of the joint surface is mainly based on fractal geometry theory, Majumdar and Bhushan [2] presented the famous M-B fractal contact model, which can calculate the normal load and total real contact area based on fractal geometry. Many researchers tried to improve the classical M-B fractal model, Wang and Komvopoulos [3-4] improved some shortcomings of M-B model, and divided the deformation of asperity into the totally elastic stage, the elastic-plastic stage and the totally plastic stage, got the elastic-plastic fractal correction model. Due to the concrete is brittle material, its surface will appear broken phenomenon, the fractal theory above mentioned does not suitable for concrete - steel joint surface, this paper hold the view that deformation of asperity through three stages with the increasing load on the concrete surface, name as, elastic, plastic and crushing stage.

\section{The total real contact area}

In this paper, the machine-foundation interface is taken as study objects, this paper hold the view that deformation of asperity through three stages with the increasing load on the concrete surface, name as, elastic, plastic and crushing stage, 
when loading on the concrete surface go beyond the limit of asperity crushed, asperity will appear crushed phenomenon.

Previous studies shows that [5], according to M-B fractal theory, the size-distribution function of the truncated microcontact area $a^{\prime}$ can be defined as

$$
n\left(a^{\prime}\right)=\frac{D}{2}\left(a_{l}\right)^{D / 2}\left(a^{\prime}\right)^{-(D+2) / 2}
$$

Where $a_{l}$ is the truncated area of the largest microcontact.

By a lot of experiments of concrete under uniaxial stresses, accepted conclusion is obtained, that is stress-strain curves for concrete under uniaxial stresses have an obvious regular characteristics. At the initial compressed stage, stress approximate follows linear with strain at this stage, the end point of this stage is called the proportional limit, the critical stress of proportional limit $\sigma_{c}=0.408 \sigma_{0}$. Then the load continue to increase until exceeds the proportional limit, the asperity started to transform into plastic deformation stage, the asperity will be crushed when the load exceeds the critical crushing stress $\sigma_{u}=0.902 \sigma_{0}$. A sharp decline in the stress-strain curve due to the crushed asperity can't bear the load,

Scholars from various countries made many prediction model based on a large number of experiments, in which CEB-FIP [6] proposed the stress-strain curve of concrete equation has been widely used, combined the stress-strain curve and the fractal theory, the relationship between microcontact stress $\sigma$ and the truncated microcontact area $a^{\prime}$ can be obtained

$$
\sigma=\sigma_{0} \frac{N \varepsilon_{0} G^{(D-1)} a^{(2-D) / 2}-G^{(2 D-2)} a^{(2-D)}}{\varepsilon_{0}^{2}+(N-2) \varepsilon_{0} G^{(D-1)} a^{\prime(2-D) / 2}}
$$

According with the equation (2), the critical area of the proportional limit $a_{c}$ and the critical crushed area $a_{u}$ can be obtained

$$
\begin{aligned}
& \sigma_{c}=0.408 \sigma_{0}=\sigma_{0} \frac{N \varepsilon_{0} G^{(D-1)} a^{\prime(2-D) / 2}-G^{(2 D-2)} a^{(2-D)}}{\varepsilon_{0}{ }^{2}+(N-2) \varepsilon_{0} G^{(D-1)} a^{\prime(2-D) / 2}} \\
& \sigma_{u}=0.902 \sigma_{0}=\sigma_{0} \frac{N \varepsilon_{0} G^{(D-1)} a^{\prime(2-D) / 2}-G^{(2 D-2)} a^{\prime(2-D)}}{\varepsilon_{0}{ }^{2}+(N-2) \varepsilon_{0} G^{(D-1)} a^{\prime(2-D) / 2}}
\end{aligned}
$$

The M-B fractal contact model shows that, if the contact area of asperity $a<a_{c}$, asperity produced plastic deformation; besides it produced elastic deformation. The relationship is related to this paper, asperity will be crushed when the contact area of 
asperity $0<a_{l}<a_{c}$; if the contact area of asperity $a_{c}<a_{l}<a_{u}$, it produced plastic deformation; and if the contact area of asperity $a_{u}<a_{l}$, it produced elastic deformation.

When the largest contact area $a_{l}>a_{u}$, the two surfaces located as totally elastic contact, the real contact area $a$ can be written as $a=a^{\prime} / 2$. Then the total elastic real contact area $A_{r e}$ can be evaluated by using the size-distribution function as follows,

$$
A_{r e}=\int_{a_{u}}^{a_{l}} a \times n\left(a^{\prime}\right) d a^{\prime}=\frac{D}{4-2 D} a_{u}^{\frac{D}{2}}\left(a_{l}^{\frac{2-D}{2}}-a_{u}^{\frac{2-D}{2}}\right)
$$

When the contact area of asperity $a_{c}<a_{l}<a_{u}$, the cross-sectional area is nearly equal to the real contact area, that is $a=a^{\prime}$, then the real plastic area is evaluated as

$$
A_{r p}=\int_{a_{c}}^{a_{u}} a \times n\left(a^{\prime}\right) d a^{\prime}=\frac{D}{2-D} a_{l}^{\frac{D}{2}}\left(a_{u}^{\frac{2-D}{2}}-a_{c}^{\frac{2-D}{2}}\right)
$$

Then the total real contact area can be expressed as

$$
A_{r}=A_{r e}+A_{r p}
$$

\section{The contact load of concrete surface}

According to Hertz theory, with the perfectly elastic deformation and perfectly plastic deformation, the corresponding elastic contact load $F_{e}$ and plastic contact load $F_{p}$ can be expressed as

$$
\begin{gathered}
F_{e}=\frac{4 E^{*} r^{3}}{3 R} \\
F_{e}=\frac{2^{(9-D) / 2}(\ln \gamma)^{1 / 2} G^{(D-1)} E^{*}\left(a^{\prime}\right)^{(3-D) / 2}}{3 \pi^{(3-D) / 2}} \\
F_{p}=H a^{\prime}
\end{gathered}
$$

Where the equivalent elastic modulus $E^{*}=\left[\left(1-v_{1}^{2}\right) / E_{1}+\left(1-v_{2}^{2}\right) / E_{2}\right]^{-1}, v_{1}$, $v_{2}, E_{1}, E_{2}$ are the Poisson ratios and elastic modulus of two surfaces respectively, $H$ is the hardness of the softer material.

According with equation (3) (10), the plastic contact load of concrete $F_{p}$ can be written as 


$$
F_{p}=\sigma_{0} \frac{N \varepsilon_{0} G^{(D-1)} a^{(4-D) / 2}-G^{(2 D-2)} a^{\prime(3-D)}}{\varepsilon_{0}^{2}+(N-2) \varepsilon_{0} G^{(D-1)} a^{(2-D) / 2}}
$$

In this paper, consider that the crushed contact load of concrete $F_{u}$ is constant at the status of crushed instant, and it is proportional to truncated microcontact area,

$$
F_{u}=\sigma_{u} a^{\prime}
$$

According to the size-distribution of truncated microcontact area, the total normal contact load $F_{a}$ can be obtained by adding the integral of normal contact load in different stages of deformation,

$$
F_{a}=\int_{a_{u}}^{a_{l}} F_{e} n\left(a^{\prime}\right) d a^{\prime}+\int_{a_{c}}^{a_{u}} F_{p} n\left(a^{\prime}\right) d a^{\prime}+\int_{0}^{a_{c}} F_{u} n\left(a^{\prime}\right) d a^{\prime}
$$

\section{Simulation and Discussion}

Based on the total real contact area model of concrete-steel joint interface, a MATLAB simulation is done by using a contact model between two rough surfaces as fig. 1 with materials (Concrete C60 and steel 45).

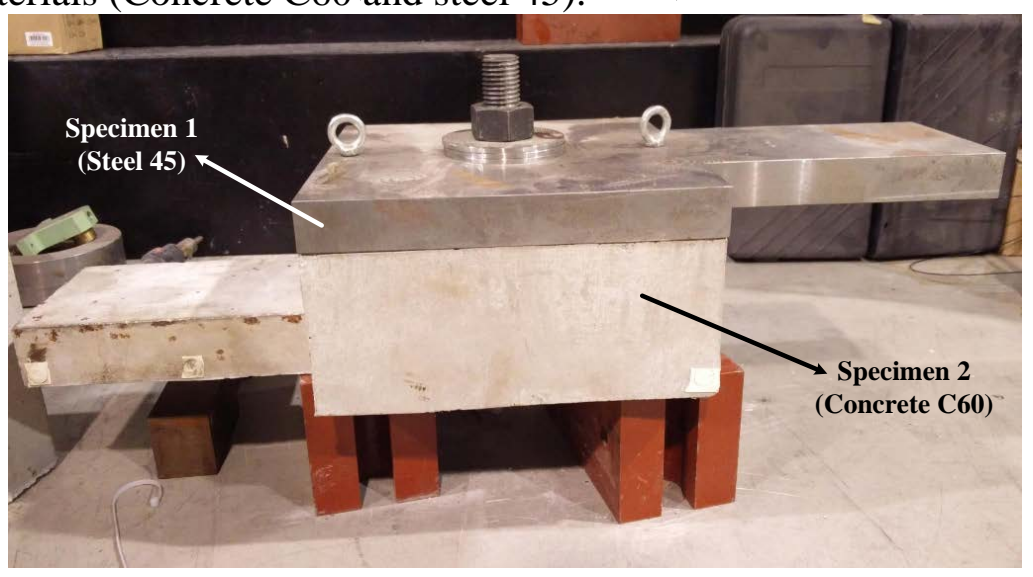

Fig.1: A contact model between two rough surfaces

Properties as table 1 assembled by using a bolt. The sample length is set as $\mathrm{L}=0.001 \mathrm{~m}$, and the topography parameter $\mathrm{G}$ and the fractal dimension $\mathrm{D}$ of the joint surface is separately as $G=9.019 \mathrm{e}-11 \mathrm{~m}, \mathrm{D}=1.284$, the results revealed the relationship of the real contact area and normal contact load of concrete-steel joint interface.

Table1: The joint surface parameters

\begin{tabular}{lll}
\hline parameters & Specimen $1(45$ steel) & Specimen 2 (concrete c60) \\
\hline Elasticity modulus & $2.06 \times 10^{11} \mathrm{pa}$ & $3.6 \times 10^{10} \mathrm{pa}$ \\
Passion ratio & 0.3 & 0.3 \\
Density & $7800 \mathrm{~kg} / \mathrm{m}^{3}$ & $2400 \mathrm{~kg} / \mathrm{m}^{3}$ \\
\hline
\end{tabular}


Fig. 2 shows that the non-linear relationship of the total normal contact load and the total contact area, and with the increase of total normal contact load, the real contact area is increased. This phenomenon can be interpreted as when the normal load applied between the rough surfaces is larger, more asperities produced contact, the total real contact area is larger, which shows that higher contact load can make the contact quality of the joint surface better,

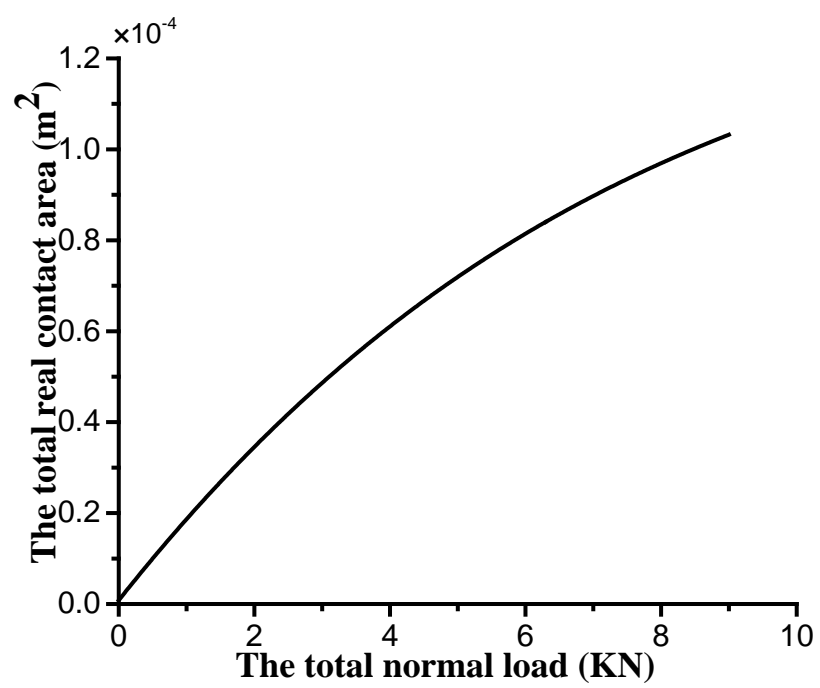

Fig.2 Relationship of the total real contact area and normal load

Fig. 3 is the size of the total real contact area of different fractal dimension D and topography parameter $G$ with the same normal load (6KN). Fig.3 (a) shows that the fractal dimension is larger, the influence of the fractal dimension on the quality of the joint surface is greater. Fig.3 (b) shows that the real contact area is decreased with the increase of the topography parameter G. From above all, the results show that t higher contact load and smoother surface (larger fractal dimension D and smaller topography parameter G) are helpful in improving the contact quality of the concrete-steel joint surface.

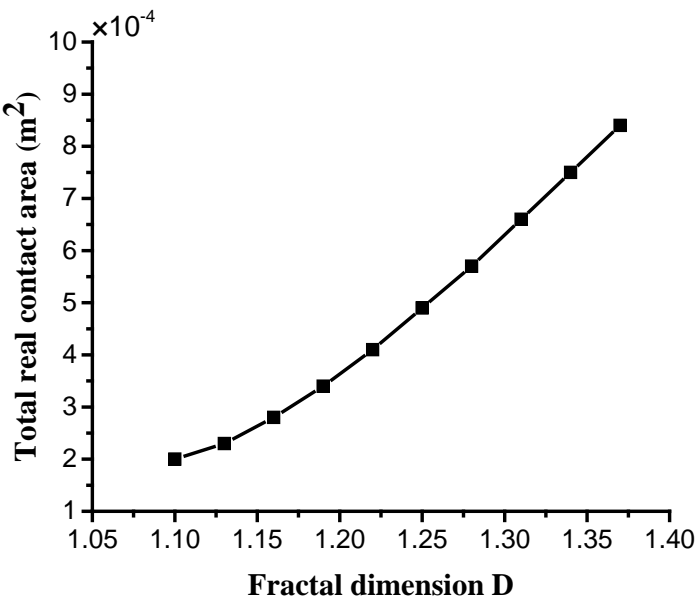

(a) $\mathrm{G}=9.019 \mathrm{e}-11 \mathrm{~m}$

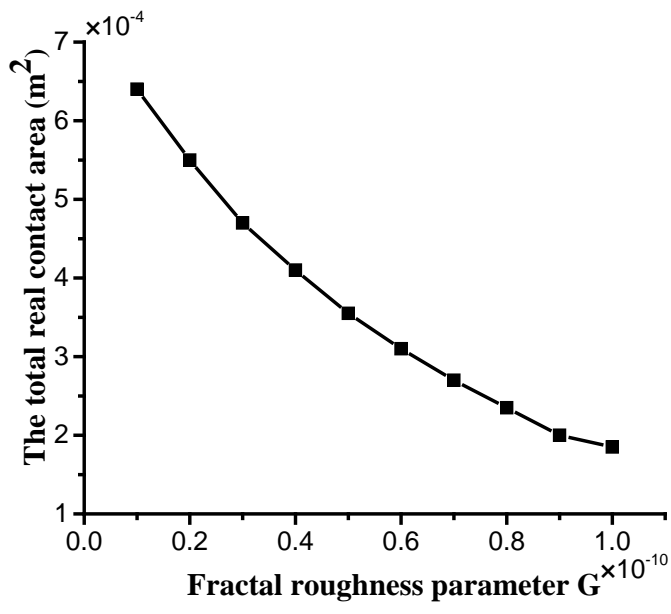

(b) $\mathrm{D}=1.284$

Fig.3 The total real contact area under different fractal parameters 


\section{Conclusion}

(1) The fractal load and contact area model of concrete-steel joint interface with considering crushed deformation are established in the paper. The MATLAB simulation with a sample is done. The result shows that the calculated real contact area based on the fractal model is closer to the actual value.

(2) The relationship between the total real contact area and the normal contact load is revealed, which shows that higher contact load can make the contact quality of the joint surface better.

(3) The research about the influence of fractal dimension and fractal roughness parameter on the real contact area illustrates that smoother surface (larger fractal dimension $\mathrm{D}$ and smaller topography parameter $\mathrm{G}$ ) are helpful in improving the contact quality of the concrete-steel joint surface.

\section{Acknowledgement}

This research was financially supported by National Natural Science Foundation of China (No.51375025), National Science and Technology Major Projects (NO.2013ZX04013-011 and NO.2015ZX04014021).

\section{References}

[1] Marco Paggi, Michele Ciavarella. The coefficient of proportionality k between real contact area and load with new asperity models. Wear, 268 (2010) 1020-1029.

[2] MAJUMDAR A,Bhushan B.Fractal model of elastic-plastic contact between rough surfaces $[\mathrm{J}]$. ASME Journal of Tribology,1991, 113(1) : 1-11.

[3] Wang S, Komvopoulos K. A fractal theory of the interfacial temperature distribution in the slow sliding regime.Part I: elasticcontact and heat transfer analysis [J]. ASME J Tribology, 1994, 116: 812-823.

[4] Wang S, Komvopoulos K A fractal theory of the interracial temperature distribution in the slow sliding regime:part I-elastic contact and heat transfer analysis.Journal of Tribology, Transactions of ASME 1994, 116(4): 812-823.

[5] Ji C C, Zhu H, Jiang W Fractal Prediction Model of Thermal contact conductance of Rough surfaces. Chinese Journal of Mechanical Engineering 2012, 26(1):128-136.

[6] Mahdi Nematzadeh, Abolghasem Salari. Stress-strain behavior of freshly compressed concrete under axial compression with a practical equation[J]. Construction and Building Materials, 2016,115(402-423). 